\title{
Line X-ray emission from Al targets irradiated by high-intensity, variable-length laser pulses
}

\author{
J. LIMPOUCH, ${ }^{1}$ O. RENNER, ${ }^{2}$ E. KROUSKÝ,${ }^{2}$ I. USCHMANN,${ }^{3}$ E. FÖRSTER, ${ }^{3}$ \\ M. P. KALASHNIKOV, ${ }^{4}$ AND P. V. NICKLES ${ }^{4}$ \\ ${ }^{1}$ Czech Technical University in Prague, Faculty of Nuclear Sciences and Physical Engineering, \\ Brehová 7, 11519 Prague, Czech Republic \\ ${ }^{2}$ Institute of Physics, Academy of Sciences CR, Na Slovance 2, 18221 Prague, Czech Republic \\ ${ }^{3}$ Institut für Optik und Quantenelektronik, Friedrich-Schiller-Universität, Max-Wien-Platz 1, 07743 Jena, Germany \\ ${ }^{4}$ Max-Born-Institut, Rudower Chaussee 4, 12489 Berlin, Germany
}

(ReCeIved 6 April 2001; ACCEPTEd 8 October 2001)

\begin{abstract}
The absolutely calibrated $K$-shell spectra emitted from short-living aluminum plasmas at laser intensities of $5 \times$ $10^{15}-4 \times 10^{18} \mathrm{~W} / \mathrm{cm}^{2}$ are reported. The experiments performed with the constant energy, variable-length laser pulses (1.5 ps-1 ns) are modeled by the one-dimensional (1D) hydrodynamics code, including nonlinear resonance absorption of the laser radiation, fast electron acceleration, and energy transfer into the target. The characteristic features of the measured and the postprocessed spectra are outlined. The spatial and temporal profiles of the emitted spectra are presented; the scaling rules for the conversion efficiency of the laser radiation into the line X-ray emission are discussed.
\end{abstract}

Keywords: Laser-produced plasma; Line X-ray emission; X-ray sources; X-ray spectroscopy

\section{INTRODUCTION}

The current interest in plasmas generated by high-intensity, ultrashort laser pulses (Gibbon \& Förster, 1996) is motivated by their relevance for laser-driven fusion experiments and a number of diverse applications, mostly related to their unique radiative properties. In rapidly evolving plasmas, the characteristic times of different atomic processes are comparable with time scales on which temperature and density changes occur. Consequently, for the laser pulse lengths at the level of tens or units of picoseconds, the plasma emission cannot be interpreted as a series of steady-state spectra (Riley, 1998). Also, the standard particle-in-cell (PIC) codes, commonly used for the description of laser absorption, are not fully adequate to model overdense plasmas, where the prevailing part of X-ray emission is generated. Thus the development of the effective and compact X-ray sources for temporally resolved measurements requires a systematic scaling of the experiments and a comparison of the experimental results with hybrid theoretical models.

Address correspondence and reprint requests to: J. Limpouch, Faculty of Nuclear Sciences and Physical Engineering CTU, Brehová 7, 11519 Prague, Czech Republic. E-mail: limpouch@ siduri.fjfi.cvut.cz
In this paper, the $\mathrm{X}$-ray line emission from solid $\mathrm{Al}$ targets irradiated by high-intensity, variable-length laser pulses is studied. We briefly describe the experimental setup used to measure the absolutely calibrated $K$-shell spectra and the conversion efficiencies of the laser energy into the line X-ray radiation. Then we provide the basic information about the theoretical modeling and discuss the conditions at which a satisfactory agreement between the observed and simulated spectra can be expected. The experimental data are compared with the results of simulations, and conclusions about spatial and temporal profiles of X-ray emission are deduced.

\section{EXPERIMENT}

The experiments were carried out by using the CPA picosecond Nd-glass laser system (wavelength $1.053 \mu \mathrm{m}$, ASE contrast $10^{9}$ ) at the MBI Berlin (Kalashnikov et al., 1994). The p-polarized laser light was incident onto bulk planar aluminum targets at an angle of $45^{\circ}$. By using an off-axis parabolic mirror (f/2, mirror-target distance of $150 \mathrm{~mm}$ ), the radiation was focused to a focal spot of less than $10 \mu \mathrm{m}$. The targets were mounted on a motorized translation stage; a high-magnification alignment system using the backscattered light controlled the target position within $\pm 10 \mu \mathrm{m}$. The energy of the laser pulses was kept constant $(\sim 4.3 \mathrm{~J})$, 


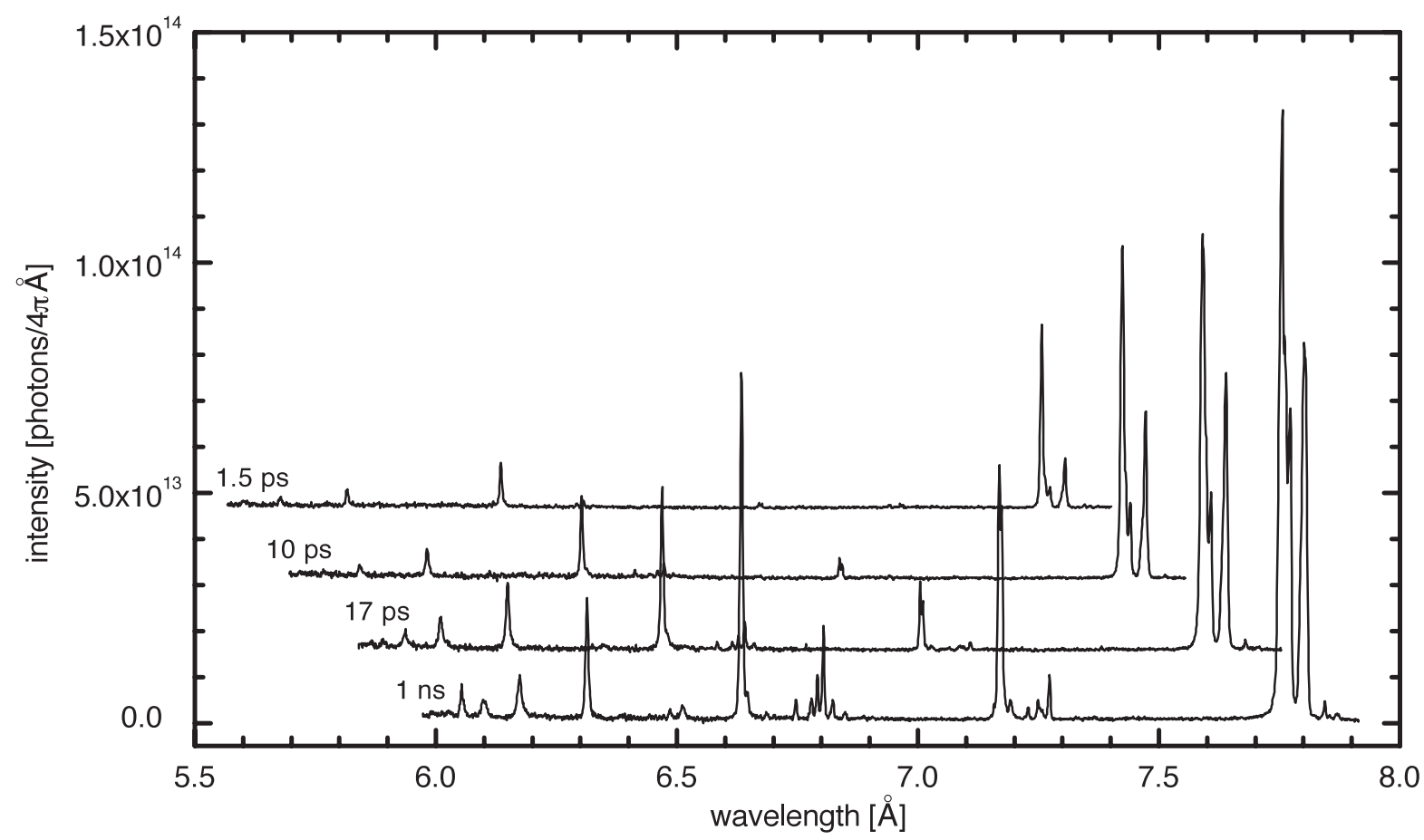

Fig. 1. Aluminum spectra recorded at laser intensities of $5 \times 10^{15} \mathrm{~W} / \mathrm{cm}^{2}$ (the FWHM laser pulse length $\tau=1 \mathrm{~ns}$ ), $3 \times 10^{17} \mathrm{~W} / \mathrm{cm}^{2}$ $(\tau=17 \mathrm{ps}), 6 \times 10^{17} \mathrm{~W} / \mathrm{cm}^{2}(\tau=10 \mathrm{ps})$, and $4 \times 10^{18} \mathrm{~W} / \mathrm{cm}^{2}(\tau=1.5 \mathrm{ps})$.

while the FWHM duration $\tau$ of the Gaussian laser pulses was set to $1.5 \mathrm{ps}, 10 \mathrm{ps}, 17 \mathrm{ps}$, and $1 \mathrm{~ns}$ by using different configurations of the grating compressor. Consequently, the laser intensity at the target surface spanned between $5 \times$ $10^{15}$ and $4 \times 10^{18} \mathrm{~W} / \mathrm{cm}^{2}$.

The $K$-shell emission between 6 and $7.9 \AA$ (i.e., 1570 to $2060 \mathrm{eV}$ ) was observed at the glancing angle of $20^{\circ}$ to the target. Absolutely calibrated, time-integrated spectra were recorded in single shots by using the von Hamos spectrometer fitted with a crystal of PET (002) cylindrically bent to a radius of $100 \mathrm{~mm}$. The plasma emission per unit wavelength interval and solid angle was derived by taking into account the integrated reflectivity of the crystal, the transmission through the $20-\mu \mathrm{m}$-thick beryllium and $0.9-\mu \mathrm{m}$-thick mylar protective foils, and the characteristic curve of the X-ray film Kodak DEF used.

As shown in Figure 1, the character of the observed spectra depends dramatically on the pulse length. The spectrum recorded at $1 \mathrm{~ns}$ contains well-resolved $\mathrm{He}-\alpha, \mathrm{He}-\beta$, and Ly- $\alpha$ groups consisting of resonance lines, intercombination lines, and accompanying satellites. Higher order transitions in He-like ions are clearly distinguished up to $\mathrm{He}-\epsilon$; hydrogenic Ly- $\beta$ is also observed. With the decreasing pulse duration, the $\mathrm{X}$-ray emission is generally weaker and the abundance ratios of individual spectral lines differ considerably. While the peak intensity of the $\mathrm{Al} \mathrm{He}$-like resonance line changes only moderately when going from $1 \mathrm{~ns}$ to $1.5 \mathrm{ps}$, the energy emitted within the full profile of the Ly- $\alpha$ drops by approximately two orders of magnitude. The peak intensities of He- and $\mathrm{H}$-like resonance lines are comparable at
1 ns laser pulse $\left(1.3 \times 10^{14}\right.$ and $5.5 \times 10^{13}$ photons $/ 4 \pi \AA$, respectively), whereas at shorter pulses, the emission of $\mathrm{Al}$ XII dominates, and simultaneously the Lyman series members are close to or even below the detection limit.

\section{COMPUTATIONAL MODEL}

The interactions of short-pulse high-intensity obliquely incident p-polarized laser beams with solid targets are extremely difficult for a theoretical description. One- and twodimensional (2D) PIC simulations model laser absorption and fast electron acceleration correctly, while the plasma dynamics description is possible only for an expanding plasma up to a few times the critical density (Gibbon et al., 1999). On the other hand, hydrodynamics models account for the physics of the dense target accurately, but they have to rely on phenomenological models of resonance absorption and hot electron generation. Even when tailored for short-pulse interaction (Andreev \& Limpouch, 1999; Eidmann et al., 2000), they cannot describe the strongly nonlinear interaction regime at the oblique incidence and the laser intensities $I \lambda^{2} \geq 10^{17} \mathrm{~W} / \mathrm{cm}^{2} \times \mu \mathrm{m}^{2}$ correctly. Taking into account these restrictions, a basic theoretical description of the experiment is derived via a 1D hydrodynamics code (Andreev \& Limpouch, 1999), including nonlinear resonance absorption of laser radiation, fast electron acceleration, and energy transport into the target. Adjustable code parameters are selected in such a way that hot electron spectra are similar to PIC simulations. The fraction of collisionless absorption going to hot electrons is ad hoc varied to 
match simulated X-ray spectra with the experiment. Due to the sharp laser focusing (focal spot diameter $\sim 10 \mu \mathrm{m}$ ), 1D planar geometry is strictly applicable for the laser pulse length of 1.5 ps only. For 10- and 17-ps laser pulses, the lateral dimension of the plasma corona exceeds the focal spot radius only after laser extinction. As the available 2D codes are not suited for short-pulse interactions, we use the described 1D model also for these pulse lengths.

Emission and transport of $\mathrm{X}$ rays is modeled in 1D planar geometry by using the postprocessor described by Andreev et al. (2000). The atomic physics model includes a detailed system of resonance levels for $\mathrm{Li}-, \mathrm{He}-$, and $\mathrm{H}$-like states as well as selected autoionization states important for X-ray diagnostics, thus providing the possibility to calculate the emission in satellites to $\mathrm{Ly}-\alpha$ and $\mathrm{He}-\alpha$ lines. Radiative transfer is solved together with the level populations for transitions with nonnegligible optical depth only. The synthetic spectra are calculated by integrating the radiation transfer equation along ray paths; the transverse dimensions of plasma are assumed to be limited by the focal spot diameter. Finally, the spatially and temporally resolved synthetic spectra are integrated over time and/or distance from the target.

\section{ENERGY TRANSFER VIA HOT ELECTRONS}

The absorption of p-polarized short pulses incident obliquely on solid targets is generally high $(\approx 50 \%)$. Assuming that a substantial part of the absorbed energy is confined within the plasma corona, the short pulses studied here should correlate with high corona temperature, nearly complete ionization of the aluminum plasma, and dominant line emission of the H-like ions.

At large laser intensities, the fraction of the absorbed energy transferred to fast electrons is, however, very high. For instance, this fraction can reach $90 \%$ for intensities around $10^{16} \mathrm{~W} / \mathrm{cm}^{2}$ (Andreev et al., 1999), and grows further with the laser intensity. A part of this energy is transferred to fast ions at the plasma vacuum boundary via acceleration by sheath electric field, but most of it is transported deep inside the bulk solid target, heating a thick surface layer to moderate temperatures. In the hydrodynamics simulations presented here, we introduced the scaling of hot electron temperature $T_{h}$ with laser intensity $I_{L}$ from PIC simulations by using the code of Gibbon (1994):

$$
T_{h}=100 \mathrm{keV}\left(\frac{I_{L} \lambda^{2}}{10^{17} \mathrm{~W} / \mathrm{cm}^{2} \times \mu \mathrm{m}^{2}}\right)^{1 / 2} .
$$

Here, the variation of the laser pulse length $\tau$ with laser intensity $\tau \sim I_{L}^{-1}$ was assumed. This approach is consistent with the classical scaling $T_{h} \sim\left(I_{L} \lambda^{2}\right)^{0.39} T_{c}^{1 / 4}$ (Estabrook \& Kruer, 1977) if the cold electron temperature in corona $T_{c} \sim$ $I_{L}^{0.44}$. For ultrashort laser pulses, Gibbon and Förster (1996) deduced hot electron temperature scaling $T_{h} \sim\left(I_{L} \lambda^{2}\right)^{1 / 3}$ from analysis of many published experiments, while a more rapid growth of hot electron temperature $T_{h} \sim\left(I_{L} \lambda^{2}\right)^{2 / 3}$ was used by Andreev et al. (1999). In previous experiments performed by using 2-ps laser pulses (Schnürer et al., 1995), the hot electron temperature scaling as high as $T_{h} \sim I_{L}^{1.4}$ was found in the intensity range $10^{17}-5 \times 10^{17} \mathrm{~W} / \mathrm{cm}^{2}$ via the measurement of hard X-ray emission from $\mathrm{Al}$ and $\mathrm{Au}$ targets. According to our experience, the applied scaling represents the best approximation for the current experimental conditions. The different methods mentioned above provide slightly different answers, but should not qualitatively change the dependence of the Ly to He resonance line ratio on the laser pulse length.

Energetic electrons penetrate deep into thick solid targets. The mass stopping range $\rho l_{h}$ of hot electrons in a solid grows according to $\sim T_{h}^{2} / \ln \Lambda_{f}$, where the effective Coulomb logarithm $\ln \Lambda_{f}$ increases very slowly with the hot electron temperature. To evaluate this parameter, an approximate formula deduced by Harrach and Kidder (1981) for an electron energy range of 1 to $200 \mathrm{keV}$ was applied. The stopping range $l_{h}$ of electrons in massive aluminum with the density $\rho_{0}=2.7 \mathrm{~g} / \mathrm{cm}^{3}$ takes the form

$$
l_{h}=69 \mu \mathrm{m} \times\left(\frac{T_{h}}{100 \mathrm{keV}}\right)^{1.72} .
$$

This stopping range is purely due to a single electron interaction with a solid matter. In high-intensity laser-plasma interactions, the hot electron flux may be so large that a strong electric field driving the return electric current into corona is generated via an accumulated spatial charge. This electric field can substantially reduce the range of hot electrons penetrating into the solid. A strong magnetic field formed by streaming particles also limits the angular spread of the fast electrons, so that they travel approximately perpendicularly to the target surface. Simple estimates and numerical simulations (Davies et al., 1997) show that the reduction of the hot electron range is substantial at intensities of $10^{18} \mathrm{~W} / \mathrm{cm}^{2}$ and above. Thus Equation (2) overestimates the electron range for the 1.5-ps laser pulse.

The energy deposition via hot electrons has a broad peak near the maximum of the electron range (Davis et al., 1995). For simplicity, our analytical model assumes a homogeneous heating of the target surface within the full stopping range of electrons. The difference between electron and ion temperatures in the dense target layer heated by hot electrons can be neglected; their values follow from the law of energy conservation:

$$
I_{a f} \tau=\left(Z_{a v}+1\right) n_{i} k_{B} T_{s} l_{h},
$$

where $I_{a f}=\eta_{f} I_{L}$ is the density of the energy flux entering the dense target layer, $\eta_{f}$ is the efficiency of the laser conversion to hot electrons traveling into the target, $Z_{a v}$ is the average ion charge in the target, $k_{B}$ is the Boltzmann constant, and $n_{i}$ is the ion density. Using scaling relations (1) and (2) we obtain temperature $T_{s}$ of the target layer heated by hot electrons: 


$$
\begin{aligned}
T_{s} & =1.5 \mathrm{keV} \frac{\eta_{f}}{\left(Z_{a v}+1\right) \lambda_{\mu}^{1.72}}\left(\frac{I_{L}}{10^{17} \mathrm{~W} / \mathrm{cm}^{2}}\right)^{0.14}\left(\frac{\tau}{1 \mathrm{ps}}\right) \\
& \cong 90 \mathrm{eV}\left(\frac{\tau}{1 \mathrm{ps}}\right)^{0.86},
\end{aligned}
$$

where $\lambda_{\mu}$ is the laser wavelength in micrometers. The conditions of our experiment may be approximated by the absorption $\eta_{f}=0.4$, average ionization $Z_{a v}=11$, corresponding to He-like ions of Al, and a constant laser fluence $I_{L} \tau=$ $6 \times 10^{6} \mathrm{~J} / \mathrm{cm}^{2}$. Then the derived scaling (4) predicts $T_{s} \approx$ $130 \mathrm{eV}, 650 \mathrm{eV}$, and $1000 \mathrm{eV}$ for $1.5 \mathrm{ps}, 10 \mathrm{ps}$, and $17 \mathrm{ps}$ pulses, respectively. The target temperature increases significantly with the laser pulse length and consequently more Al ions are ionized up to H-like and fully stripped states. Direct ionization by fast electrons is negligible as the ionization cross section reduces considerably at fast electron energies above the ionization potential. Thus our simplified model qualitatively explains the observed rise of the Lyman to He-like resonance line ratio with the increasing laser pulse duration. This behavior is further strengthened by time-limited occurrence of high electron temperatures in short-pulse experiments.

The physics of the interaction of the nanosecond laser pulse with the target is substantially different. The fraction of the energy absorbed by fast electrons is considerably lower. Their characteristic temperature $T_{h}$ amounts to $10 \mathrm{keV}$, and thus their stopping range is shorter than the electron temperature scale length. Accordingly, the dense target material is basically heated by an electron thermal wave propagating from corona to the target. The laser intensity of $10^{15} \mathrm{~W} / \mathrm{cm}^{2}$ is high enough to produce electron temperatures of the order of $1 \mathrm{keV}$ in the dense plasma between the critical and the ablation surface. At these conditions, expanding plasma is ionized to H-like or fully stripped ions and Lyman line is comparable to the He-like resonance line emission. This case is basically two-dimensional, as discussed in detail elsewhere (Renner et al., 2001).

\section{RESULTS AND DISCUSSION}

The hydrodynamic simulations performed cannot reliably calculate the fraction of the absorbed laser energy transferred to fast electrons. This fraction was therefore varied in order to improve the fit of simulated results with the experiment. The simulations indicate that the integral energy of expanding plasma corona contains a relatively small fraction of the absorbed laser energy $(\approx 4 \%, 3 \%$, and $0.7 \%$ for 17-ps, 10-ps, and 1.5-ps laser pulses, respectively), which is, however, considerably higher than collisional laser absorption. The spatial profiles of the plasma electron temperature and density at the laser pulse maximum are displayed in Figure 2a for various laser pulse lengths. In agreement with the discussion of Equation (1), the temperature of the subcritical density corona is higher for shorter laser pulses. We note that there is a great deal of uncertainty in maximum
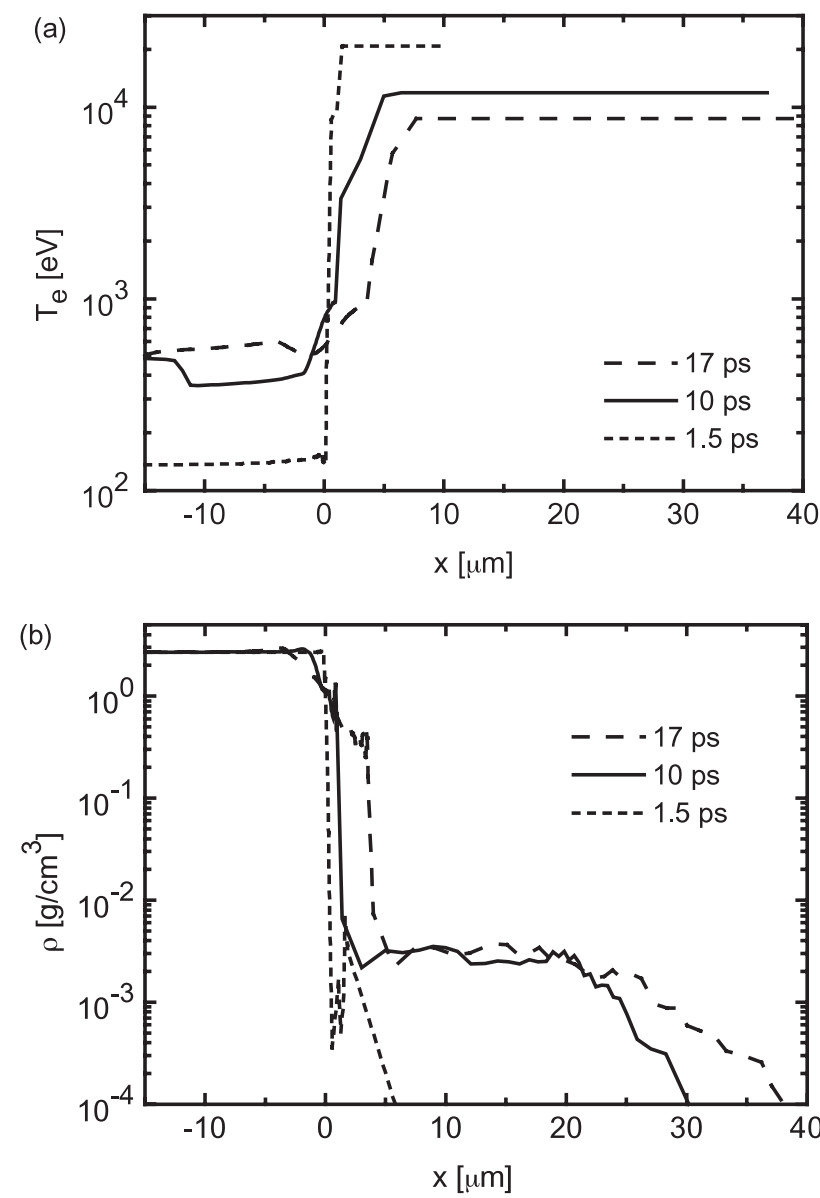

Fig. 2. Spatial profiles of the electron temperature (a) and plasma density (b) at the maximum of the 1.5-ps, 10-ps, and 17-ps laser pulses. The laser intensities are the same as in Figure 1; the position of the fresh target surface is defined by $x=0$.

corona temperature as it depends on many factors such as heat flux limiter that are described only qualitatively in hydrodynamics models. However, its exact value is insignificant here as the contribution of the underdense corona to the line X-ray emission is negligible. In contrast, the temperature of the overdense plasma grows with the laser pulse length as predicted by Equation (4). The propagation of the thermal wave into the dense material contributes to additional heating of the target surface. The density profile steepening by ponderomotive force limits the mass of underdense corona, as shown in Figure $2 \mathrm{~b}$.

The time-dependent hydrodynamics parameters are postprocessed by using the atomic physics package (Andreev et al., 2000). The results obtained provide information about the spatial and temporal distribution of the line emission, which is substantial for different sort of applications. The spatially resolved synthetic X-ray spectra are presented in Figure 3. For the 1.5-ps laser pulse, the line emission peaks at $4 \mu \mathrm{m}$ and vanishes at about $10 \mu \mathrm{m}$ from the target surface. For the 17-ps laser pulse, the calculated extent of the line-emitting region is approximately $60 \mu \mathrm{m}$ from the target 

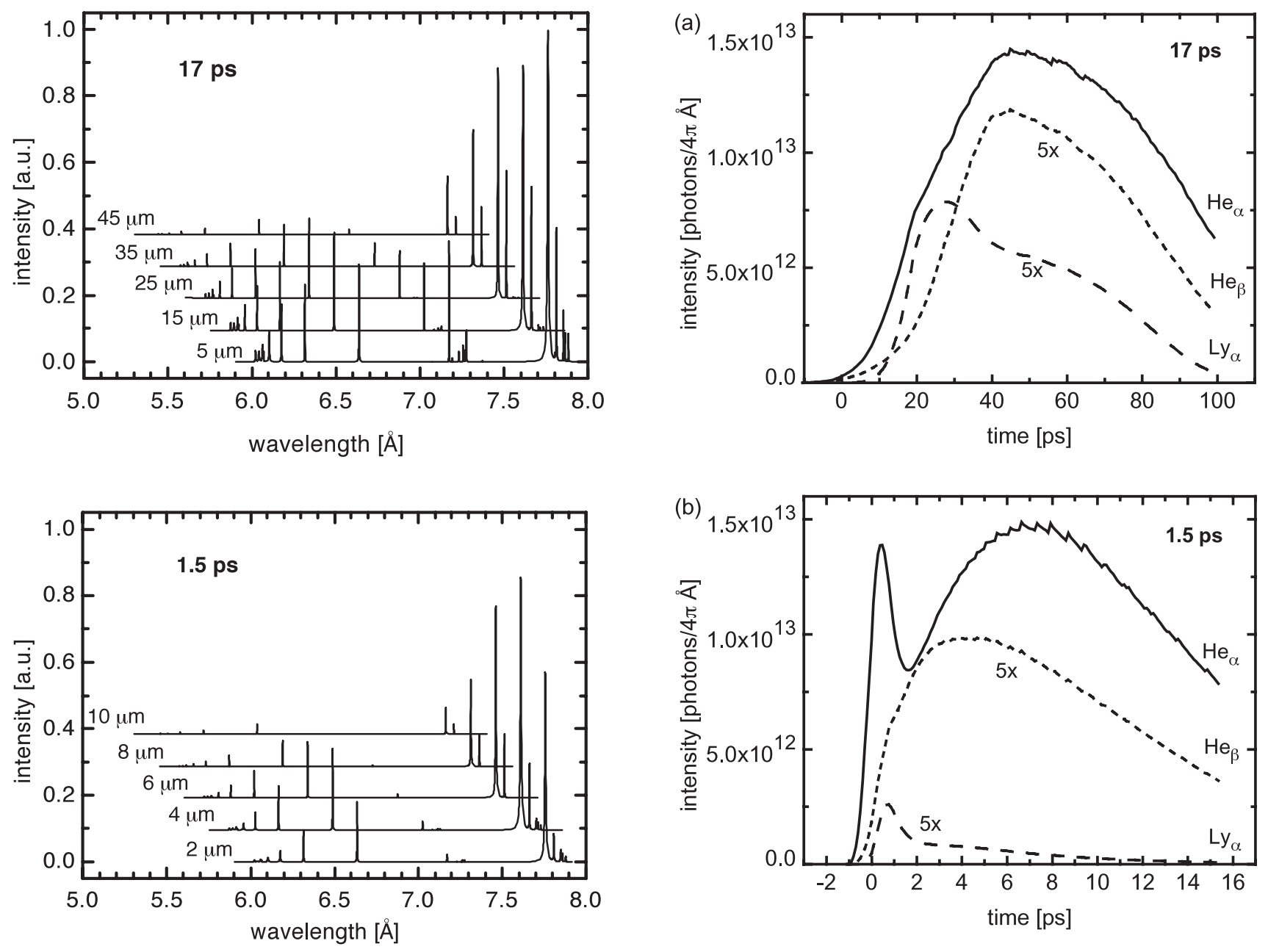

Fig. 3. Computational X-ray spectra as a function of the distance from the target surface (the model does not include $\mathrm{He}-\beta$ satellites). Simulations performed for the laser pulse lengths of $17 \mathrm{ps}$ and $1.5 \mathrm{ps}$ demonstrate the variable spatial extent of the observable X-ray emission.

surface. This agrees well with our spatially resolved measurements (Renner et al., 2001) where the Ly- $\alpha$ emission was observed up to the distance of $70 \mu \mathrm{m}$ from the target. The comparison of simulated profiles of the He- $\alpha$ resonance and intercombination line demonstrates clearly the effects of reabsorption. If the reabsorption is fully switched off, the intensity in the center of the He- $\alpha$ line increases by one order of magnitude or even more and the total emission in lines is overestimated. When neglecting only the effect of reabsorption on energy level populations, that is, assuming optically thin populations, the line emission decreases by less than $50 \%$. The calculated emission spectra agree well with the experiment in energy ratio of dominant resonance lines and also in the intercombination to $\mathrm{He}-\alpha$ line ratio. The relative intensities of satellites to resonance lines are of the same order of magnitude as experimentally observed values.

The temporal profiles of the spatially integrated X-ray emission in dominant lines are plotted in Figure 4. For the 1.5 -ps laser pulse, the nonstationary character of the He- $\alpha$

Fig. 4. Temporal profiles of the He- $\alpha$, He- $\beta$, and Ly- $\alpha$ pulses emitted from plasma heated by 17 -ps (a) and 1.5-ps (b) laser pulses. The zero time coordinate corresponds to the pulse maximum; intensities of the $\mathrm{He}-\beta$ and Ly- $\alpha$ emission are multiplied by a factor of 5 .

emission is particularly obvious. A narrow minimum predicted about 2 ps after the laser pulse maximum can be ascribed to transient ionization to H-like state. The calculated intensities of emitted He-like resonance lines are comparable for both cases, but the duration of X-ray pulses grows with the laser pulse length. Figure $4 \mathrm{~b}$ also indicates that for the 1.5-ps laser pulse, the duration of Ly- $\alpha$ emission is comparable with the laser pulse length (the He-like emission is approximately 10 times longer), and consequently this line is attractive as a potential ultrashort probing source for various applications.

The measured and calculated efficiencies of the laser energy transformation into the emission of $\mathrm{He}-\alpha$ and $\mathrm{Ly}-\alpha$ lines are plotted in Figure 5 as a function of the laser pulse length. The values computed for the 1-ns laser pulse using the described methodology are displayed for completeness only, the adequate description of this experiment requires 2D simulations (Renner et al., 2001). The theoretical and experimental values agree within the factor of 2 with the 


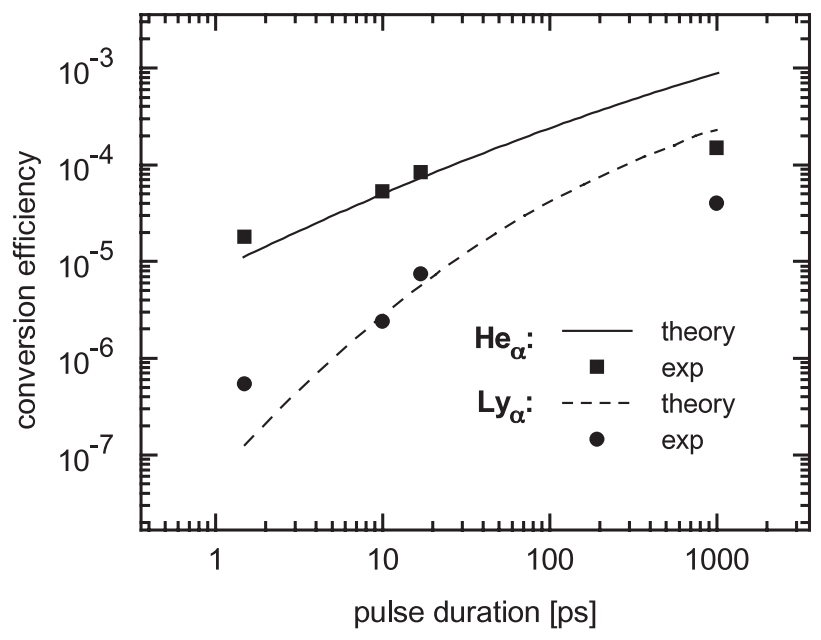

Fig. 5. Simulation and experimental conversion efficiencies of the laser energy into the emissions of the $\mathrm{He}-\alpha$ and Ly- $\alpha$ spectral lines.

exception of the 1-ns laser pulse. By using the experimental data, we have deduced a scaling law for the energy conversion efficiency $\eta$. At the laser pulse length $\tau$ below $17 \mathrm{ps}$, the scaling is $\eta \sim \tau^{0.6}$ for the He-like $(\alpha, \beta)$ resonance lines and $\eta \sim \tau$ for the H-like emission. For longer laser pulses, the relations are weaker; $\eta \sim \tau^{0.15}$ for the He-like lines and $\eta \sim$ $\tau^{0.4}$ for the Ly- $\alpha$ line. As mentioned above, a satisfactory agreement between the observed and simulated spectra could only be achieved assuming that the prevailing part of the absorbed laser energy is transferred by very hot electrons outside the interaction region, that is, deeper into the target. Despite approximations introduced into the hydrodynamics model, we believe it can provide a sufficiently detailed insight into the physics of the experiments and explain qualitatively the emission characteristics observed.

\section{CONCLUSIONS}

We present a systematic study of the line X-ray emission from aluminum targets irradiated by high-intensity, variablelength laser pulses. The absolutely calibrated single-shot $\mathrm{X}$-ray spectra from plasma generated by obliquely incident p-polarized laser light display a distinct redistribution of the emitted energy as a function of the pulse duration. The observed high intensities of the emitted spectral lines offer interesting possibilities for development of monochromatic $\mathrm{X}$-ray sources synchronized to the laser pulse.

For the laser pulse lengths up to $17 \mathrm{ps,} \mathrm{the} \mathrm{experimental}$ spectra were successfully simulated by using 1D hydrodynamics code tailored for short pulse interactions. The simulations indicate that a dominant fraction of laser energy is transferred to fast electrons. The experiments were performed at the constant laser fluence $I_{L} \tau$, and thus approximately the same amount of energy was deposited into the target. The hot electron temperature $T_{h}$ grows with the laser intensity $I_{L} \lambda^{2}$ and simultaneously the electron stopping range $l_{h}$ increases. Thus for higher intensities and shorter laser pulses, the mass heated by fast electrons is larger and the predicted temperature of this layer decreases; consequently the abundance of the excited H-like ions is smaller. This plasma behavior explains the dominant features of the observed time-integrated spectra. The line emission calculated via atomic physics and radiation transport postprocessor, particularly the intensities and ratios of resonance lines, He-like intercombination line, and Li-like satellites, agree reasonably well with observations.

Despite the limitations of the adopted theoretical model, we believe the simulations provide realistic information about the spatial and temporal distribution of X-ray line emission. The favorable comparison of the measured and simulated spectra, including the conversion efficiency of the laser radiation into the $\mathrm{X}$-rays, indicates that the developed theory is adequate to model the radiative properties of plasmas generated by high-intensity laser pulses with picosecond or subnanosecond duration. The detailed knowledge of the emission characteristics is important for potential applications of these short pulse X-ray sources.

\section{ACKNOWLEDGMENTS}

The authors gratefully acknowledge the assistance of the scientific and technical staff from MBI Berlin and IOQ Jena. This work was supported by the Grant Agency of the Czech Republic under grant 202/01/0755 and by the EEC Large Facility Programme under contract MBI-98-04-01.

\section{REFERENCES}

Andreev, A.A. \& Limpouch, J. (1999). Ion acceleration in shortpulse laser target experiments. J. Plasma Physics 62, 179-193.

Andreev, A.A., Limpouch, J. \& Nakano, H. (2000). Optimal enhancement of X-ray line emission from plasma by shaping of short high-intensity laser pulses. Proc. SPIE 3934, 52-60.

Andreev, A.A., Teubner, U., Kurnin, I.V. \& Förster, E. (1999). Prospects of "water-window" X-ray emission from subpicosecond laser plasmas. Appl. Phys. B 69, 505-515.

Davies, J.R., Bell, A.R., Haines, M.G. \& Guérin, S.M. (1997). Short-pulse high-intensity laser-generated fast electron transport into thick solid targets. Phys. Rev. E 56, 7193-7203.

Davis, J., Clark, R. \& Guiliani, J. (1995). Ultrashort pulse laser-produced Al/Si plasma. Laser Part. Beams 13, 3-18.

Eidmann, K., Meyer-ter-Vehn, J., Schlegel, T. \& Hüller, S. (2000). Hydrodynamic simulation of subpicosecond laser interaction with solid-density matter. Phys. Rev. E 62, 1202-1214.

Estabrook, K. \& Kruer, W.L. (1977). Properties of resonantly heated electron distributions. Phys. Rev. Lett. 40, 42-45.

GibBon, P. (1994). Efficient production of fast electrons from femtosecond laser interactions with solid targets. Phys. Rev. Lett. 73, 664-667.

Gibbon, P., Andreev, A.A., Lefebre, E., Bonnaud, G., Ruhl, H., Delletrez, J. \& Bell, A.R. (1999). Calibration of onedimensional boosted kinetic codes for modelling high-intensity laser-solid interactions. Phys. Plasmas 6, 947-953. 
Gibbon, P. \& Förster, E. (1996). Short-pulse laser-plasma interactions. Plasma Phys. Control. Fusion 38, 769-793.

Harrach, R.J. \& KidDER, R.E. (1981). Simple model of energy deposition by suprathermal electrons in laser-irradiated targets. Phys. Rev. A 23, 887-896.

Kalashnikov, M.P., Nickles, P.V., Will, I., Billhardt, F. \& SCHNÜRER, M. (1994). A high-contrast all-glass ps-terawatt CPA laser system. Laser Part. Beams 12, 463-469.

Renner, O., Rosmej, F.B., Krouský, E., Sondhauss, P., KalashNikov, M.P., Nickles, P.V., Uschmann, I. \& Förster, E.
(2001). Aluminum Lyman $\alpha$ group formation at high-intensity, high-energy laser-matter interaction. J. Quant. Spectrosc. Radiat. Transfer 71, 623-634.

Riley, D. (1998). Time dependent modelling of $K$-shell emission lines from laser produced plasmas. J. Quant. Spectrosc. Radiat. Transfer 60, 221-230.

Schnürer M., Kalashnikov, M.P., Nickles, P.V., Schlegel, T., Sandner, W., Demchenko, N., Nolte, P. \& Ambrosi, P. (1995). Hard X-ray emission from intense short pulse laser plasmas. Phys. Plasmas 2, 3106-3110. 